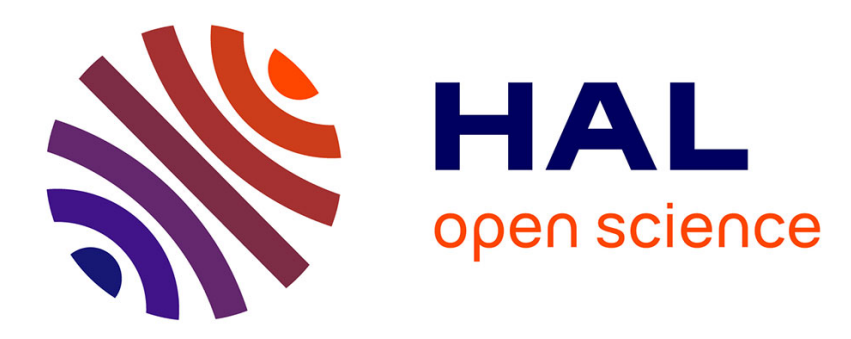

\title{
Robust Gain Scheduling techniques for Adaptive control.
}

\author{
P. Antoinette, G. Ferreres
}

\section{To cite this version:}

P. Antoinette, G. Ferreres. Robust Gain Scheduling techniques for Adaptive control.. European Control Conference, Aug 2009, BUDAPEST, Hungary. hal-01059732

\section{HAL Id: hal-01059732 \\ https://hal-onera.archives-ouvertes.fr/hal-01059732}

Submitted on 1 Sep 2014

HAL is a multi-disciplinary open access archive for the deposit and dissemination of scientific research documents, whether they are published or not. The documents may come from teaching and research institutions in France or abroad, or from public or private research centers.
L'archive ouverte pluridisciplinaire HAL, est destinée au dépôt et à la diffusion de documents scientifiques de niveau recherche, publiés ou non, émanant des établissements d'enseignement et de recherche français ou étrangers, des laboratoires publics ou privés. 


\title{
Robust Gain Scheduling techniques for Adaptive control
}

\author{
Patrice Antoinette and Gilles Ferreres
}

\begin{abstract}
Robust and gain-scheduling analysis and design techniques are used to realize an adaptive controller. LFT representations of the plant and controller are used so that the stability and performance properties of the adaptive scheme can be studied off-line, and the on-line computational time for implementing the LFT controller is reduced. This controller is scheduled as a function of the parameters to be estimated.
\end{abstract}

\section{INTRODUCTION}

A controller is said to be adaptive when it adapts to the unknown plant. Generally, measurements of input and ouput signals are used to adjust the controller. To achieve this type of control, two schemes can be identified : direct and indirect schemes. In the latter, using input and output signals of the plant, parameters of the plant model are estimated, and this estimation is used to adjust on-line the controller. The situation is described by figure 1 , where the vector of parameters $(\theta)$ is estimated $(\hat{\theta})$ thanks to the measurements of input $\left(u \in \mathbb{R}^{m_{u}}\right)$ and output $\left(y \in \mathbb{R}^{r_{y}}\right)$ signals of the plant $(H(s, \theta))$. These estimations are transmitted to the controller $(K(s, \hat{\theta}))$ to adjust it. $d \in \mathbb{R}^{m_{d}}$ is an unmeasured perturbation, and $p \in \mathbb{R}^{r_{p}}$ is a controlled output. On the contrary, in direct adaptive schemes, the controller is directly adjusted from the input and output signals of the plant, without any estimator : this is the case of the well known Model Reference Adaptive Control (MRAC). See [1] and included references. Another example is the STAC technique (Set Theoretic Adaptor Control) that is based on the concept of falsification [2], [3], [4].

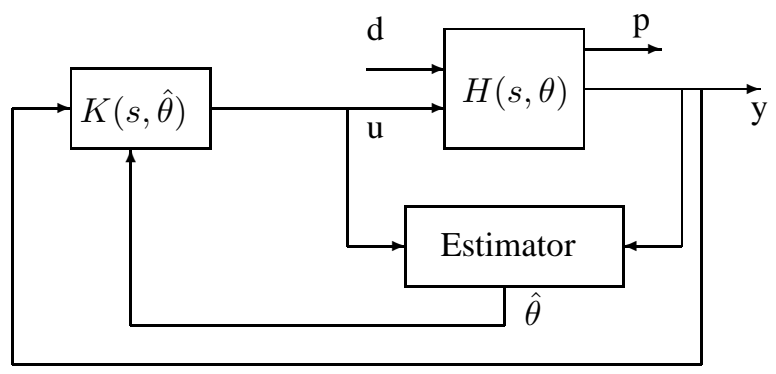

Fig. 1. General scheme of indirect adaptive control. $\theta$ is a vector of plant parameters, $\hat{\theta}$ is the estimation of $\theta, H(s, \theta)$ is the plant, and $K(s, \hat{\theta})$ is the controller.

P.Antoinette is with Onera, the French Aerospace Lab, Toulouse Research Center, BP 4025 - 31055 Toulouse Cedex 4 - France and with the Institut Supérieur de l'Aéronautique et de l'Espace (ISAE), the French aerospace engineering school. patrice.antoinette@onera.fr

G.Ferreres is with Onera, the French Aerospace Lab, Toulouse Research Center, BP 4025 - 31055 Toulouse Cedex 4 - France. gilles.ferrereseonera.fr
Results now exist on the key stability and robustness issues of adaptive control, see e.g. [5], [1], [6], [7]. To some extent, our technique could be considered as reminiscent of the theoretical framework proposed in [5]. It is especially possible to use Lyapunov and passivity techniques extracted from nonlinear analysis tools, i.e. the adaptive controller is considered as a nonlinear one [8], [9]. Despite their popularity, the application to adaptive control of linear robust control techniques ( $H_{\infty}$ design and $\mu$ analysis) and of gainscheduled control (LPV methods) has been (much) less investigated, see for instance [10], [11].

It is worth emphasizing that several issues can be studied, namely the mere asymptotic stability of the adaptive scheme or its performance, i.e. the quality of its transient (before the estimator converges in the context of indirect adaptive control) or asymptotic responses. It is also possible in some cases to prove the finite-time convergence of the direct or indirect adaptive scheme [2], [3], [10], [1]. Noting that controlling the transient response is a key issue in practice, otherwise the plant (e.g. an airplane) could be endangered.

In the present article, only indirect adaptive control of linear time invariant plants is dealt with. As a consequence, the vector $\theta$ is constant. The approach is distinguished by the fact that the estimator doesn't "physically" appear in the analysis and synthesis schemes. Only design features on the estimation errors are considered.

The article is organised in 7 sections. The problem is stated in section II. Then synthesis (III) and validation (IV) methods for an adaptive controller are described : a modal LFT gain design and a robustness analysis techniques extracted from [12], [13] are used. Section V deals with the estimation of a continuous state space representation with a standard recursive least squares algorithm [14]. All these methods set out are then applied to a transport aircraft in section VI, before the conclusion in section VII.

\section{Problem Statement}

During the realization of an indirect adaptive controller it is essential to face up to two crucial problems. The first is the on-line computational time of the controller implementation which must be reasonable. The second is to ensure closed loop stability and performance. In fact, the parameters are not directly measured but estimated. This estimation introduces a difference $\delta \theta=\hat{\theta}-\theta \neq 0$. This difference is produced by errors during the transient and steady states of the estimator. The goal is to ensure stability and "satisfactory" performance despite this error. So the controller must be robust in the face of estimation errors of $\theta$, and other modeling errors.

In order to solve these problems, the scheme presented on 
figure 2 is proposed, where the controller and the plant are both under an LFT form.

NotATIONS : In the following, notations are defined by $\theta=\left[\theta_{1}, \ldots, \theta_{n}\right]^{T}, \hat{\theta}=\left[\hat{\theta}_{1}, \ldots, \hat{\theta}_{n}\right]^{T}$ and $\delta \theta=\left[\delta \theta_{1}, \ldots, \delta \theta_{n}\right]^{T}$. Without loss of generality, it is supposed that $\theta_{i} \in[-1,1]$. Then it is assumed that $\Delta_{h}(\theta)=\operatorname{diag}\left(\theta_{1} I_{h_{1}}, \ldots, \theta_{n} I_{h_{n}}\right)$ and $\Delta_{k}(\hat{\theta})=\operatorname{diag}\left(\hat{\theta}_{1} I_{k_{1}}, \ldots, \hat{\theta}_{n} I_{k_{n}}\right)$, with integers $h_{1}, \ldots, h_{n}$, $k_{1}, \ldots, k_{n}$. Let

$$
\tilde{\Delta}=\operatorname{diag}\left(\theta_{1} I_{q_{1}}, \ldots, \theta_{n} I_{q_{n}}\right)
$$

be the matrix obtained by merging and ordering the elements of matrices $\Delta_{h}(\theta)$ and $\Delta_{k}(\theta)$, with $q_{i}=h_{i}+$ $k_{i}$, and $B o=\left\{\tilde{\Delta}(\theta) \mid \theta_{1}, \ldots, \theta_{n} \in[-1,1]\right\}, B_{\delta} o=$ $\left\{\Delta_{k}(\delta \theta) \mid \delta \theta_{1}, \ldots, \delta \theta_{n} \in[-1,1]\right\}$.

It is worth emphasizing that the set of possible values of $\theta$, the true values of the plant parameters, is assumed to be a priori given. After normalization, all $\theta_{i}$ belong to the interval $[-1,1]$.

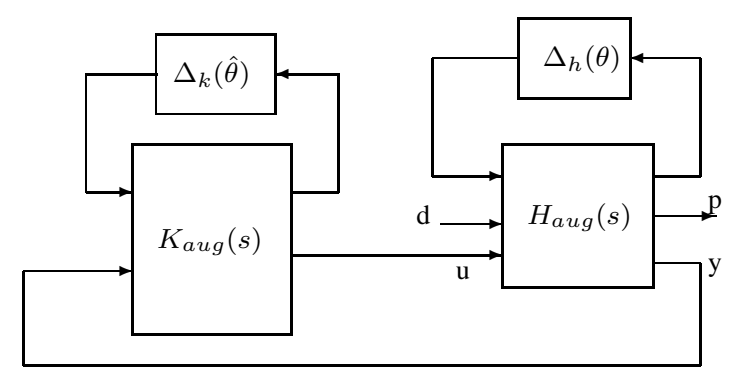

Fig. 2. Adaptive control scheme with LFT modeling. $K_{\text {aug }}(s)$ is the part of the controller designed off-line. $\Delta_{h}(\theta)$ and $\Delta_{k}(\hat{\theta})$ represent the dependence of the plant and the controller on respectively the parameter $\theta$ and its estimated value $\hat{\theta}$.

The LFT modeling presents two interests :

- First of all, thanks to this controller pattern, the computation time of the controller adjustment is expected to be reduced. In fact, in this case, the computation of $K(s, \hat{\theta})=\mathcal{F}_{u}\left(K_{a u g}(s), \Delta_{k}(\hat{\theta})\right)$ is made of two parts : $K_{\text {aug }}$ is designed off-line, and only $\Delta_{k}(\hat{\theta})$ is adjusted on-line [15].

- Futhermore, this pattern allows the off-line analysis of stability and performance properties of the adaptive closed loop with the help of robustness analysis tools [12].

In this scheme, the adaptive controller is a set composed of an estimator and a gain-scheduled controller. The robustness of the controller in the face of estimation errors on $\theta$ and other modeling errors is ensured thanks to methods of robust synthesis and analysis.

To design a controller $\mathcal{F}_{u}\left(K_{\text {aug }}, \Delta_{k}(\hat{\theta})\right)$, a method in two stages is presented : the first is the synthesis of an LFT controller, and the second is its validation.

\section{GAIN SCHEDULING CONTROL DESIGN}

\section{A. Synthesis of the LFT gain}

Contrary to the assumptions made later for the analysis, during the synthesis, the Certainty Equivalence principle is applied, and it is considered that $\hat{\theta}(t)=\theta$ for all $t$. Then a gain scheduled controller synthesis algorithm presented in [13] is used.

Proposition 1 (Algorithm of modal design): Let $(A(\theta)$, $B(\theta), C(\theta), D(\theta))$ be a state space representation, with $n_{s}$ states and $r_{y}$ outputs. If the number of measurements is greater or equal than the number $r_{d}$ of dominant modes the following algorithm can be proposed :

1) Choose $r_{d}$ closed loop eigenvalues $\lambda_{i}(\theta)$.

2) Compute $r_{d}$ pairs $\left(v_{i}(\theta), w_{i}(\theta)\right)$ so that :

$$
\left[\begin{array}{ll}
A(\theta)-\lambda_{i}(\theta) I_{n_{s}} & B(\theta)
\end{array}\right]\left[\begin{array}{c}
v_{i}(\theta) \\
w_{i}(\theta)
\end{array}\right]=0
$$

3) Compute $K(\theta)$ as a solution of the equation :

$$
K(\theta)\left[\begin{array}{ll}
C(\theta) & D(\theta)
\end{array}\right]\left[\begin{array}{c}
V(\theta) \\
W(\theta)
\end{array}\right]=W(\theta)
$$

with $W(\theta)=\left[w_{1}(\theta), \ldots, w_{r_{d}}(\theta)\right]$ and $V(\theta)=$ $\left[v_{1}(\theta), \ldots, v_{r_{d}}(\theta)\right]$.

In this algorithm, $A(\theta), B(\theta), C(\theta), D(\theta), K(\theta)$ and $\lambda_{i}(\theta)$ are under an LFT form.

It's worth noting that no more than $r_{y}$ eigenvalues can be assigned. In practice, only dominant modes are assigned, and for instance the actuator modes placement is uncontrolled. So, contrary to LPV methods which offer a guarantee of robust stability, in the algorithm used, the stability of the closed loop must be checked a posteriori. But in another respect, as $\theta$ is time invariant parameter, in the modal design there is no loss of performance due to conservative stability requirements, contrary to LPV methods which deal with time varying parameters.

\section{B. Well-posedness problem}

The result of the previous synthesis is a static LFT gain $K(\theta)=\mathcal{F}_{u}\left(K_{a u g}, \Delta_{k}(\theta)\right)$, with $K_{a u g}=\left(\begin{array}{ll}K_{11} & K_{12} \\ K_{21} & K_{22}\end{array}\right)$. Then

$$
K(\theta)=K_{22}+K_{21} \Delta_{k}(\theta)\left(I-K_{11} \Delta_{k}(\theta)\right)^{-1} K_{12}
$$

The LFT gain is well-posed if and only if $\forall \Delta_{k}(\theta) \in B_{\delta} o$, $\operatorname{det}\left(I-K_{11} \Delta_{k}(\theta)\right) \neq 0$. In order to verify the wellposedness, the structured singular value is used ([16]).

Definition 1: Let $M$ be a complex matrix with the same dimensions as $\tilde{\Delta}$ in equation (1). The structured singular value $\mu(M)$, associated to $M$ and to a real perturbation $\tilde{\Delta}$ is defined as

$$
\frac{1}{\mu(M)}=\min \{k \mid \exists \tilde{\Delta} \in k B o, \text { s.t. } \operatorname{det}(I-M \tilde{\Delta})=0\}
$$

and $\mu(M)=0$ if no $\tilde{\Delta}$ satisfies $\operatorname{det}(I-M \Delta)=0$.

The well-posedness of $K(\theta)$ can be checked with the following proposition. 
Proposition 2: $\mathcal{F}_{u}\left(K_{\text {aug }}, \Delta_{k}(\theta)\right)$ is well-posed for all $\Delta_{k}(\theta) \in B_{\delta} O$ if and only if $\mu\left(K_{11}\right)<1$. A sufficient condition is that there exist scaling matrices $D_{0}=D_{0}^{*}>0$ and $G_{0}=G_{0}^{*}$, with $D_{0} \Delta_{k}(\theta)=\Delta_{k}(\theta) D_{0}$ and $G_{0} \Delta_{k}(\theta)=$ $\Delta_{k}(\theta)^{*} G_{0}$ which satisfy

$$
K_{11} D_{0} K_{11}^{*}+\jmath\left(G_{0} K_{11}^{*}-K_{11} G_{0}\right) \leqslant D_{0}
$$

Condition (6) can be checked with standard Matlab routines of the $\mu$ Analysis and Synthesis Toolbox or of the LMI Control Toolbox.

Once the well-posedness of the controller is checked, as explained previously, the next stages are to verify that the designed controller ensures the stability of the closed loop for all considered values of $\theta$, and then to compute the maximal allowable estimation error.

\section{VALIDATION WITH $\mu$-ANALYSIS}

\section{A. Building of the validation scheme}

Applying the "Certainty Equivalence" principle, so far it was supposed that $\hat{\theta}(t)=\theta$. In the following, this principle is not valid anymore and it is considered that $\hat{\theta}_{i}(t)=\theta_{i}(1+$ $\left.\delta \theta_{i}(t)\right)$, where $\delta \theta_{i}(t)$ is the relative error on parameter $\theta_{i}$. Noting that $\Delta_{k}(\hat{\theta})=\Delta_{k}(\theta)\left[I+\Delta_{k}(\delta \theta)\right]$, the scheme of figure 2 is transformed into the one of figure 3 . Then, keeping the connexions between the blocks, $H_{a u g}(s)$ and $K_{a u g}(s)$ are merged into the block $N(s)$ of figure 4 . Its first inputs are the vectors $f_{1}$ and $f_{2}$ merged and ordered following the order of $\tilde{\Delta}$, see equation (1). Next come $f_{3}$ and the unmeasured perturbation $d$. The outputs of $N(s)$ follow the same logic. Noting $\Delta_{\delta}=\Delta_{k}(\delta \theta)$, the described scheme is presented on figure 4 .

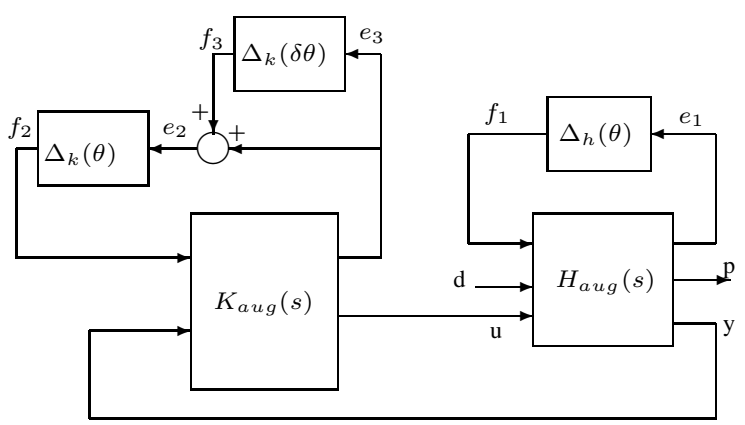

Fig. 3. Adaptive control scheme emphasizing the estimation error.

First of all, as indicated previously, in order to end the synthesis, the stability of the scheme of figure 4 whithout estimation errors (i.e $\Delta_{\delta}=0$ ) must be checked with the following proposition.

Proposition 3: Let $N_{11}(s)$ be the asymptotically stable transfer matrix between $w$ and $z$ on figure 4. Robust stability of the closed loop of figure 4 is ensured for all $\tilde{\Delta} \in B o$, with $\Delta_{\delta}=0$, if and only if $\mu\left(N_{11}(\jmath \omega)\right) \leqslant 1, \forall \omega \in[0,+\infty)$. A sufficient condition is that there exist frequency dependent scaling matrices $D_{1}(\omega)=D_{1}^{*}(\omega)>0$ and $G_{1}(\omega)=$ $G_{1}^{*}(\omega)$ which satisfy $D_{1}(\omega) \tilde{\Delta}=\tilde{\Delta} D_{1}(\omega)$ and $G_{1}(\omega) \tilde{\Delta}=$

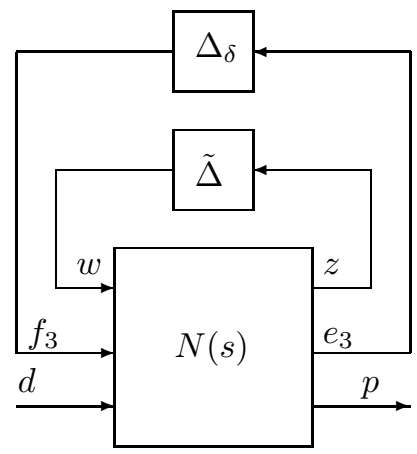

Fig. 4. Scheme used to validate the adaptive control scheme.

$\tilde{\Delta}^{*} G_{1}(\omega)$. The interconnection structure of figure 4 is stable for $\Delta_{\delta}=0$ and $\tilde{\Delta} \in B o$ if there exist $D_{1}(\omega)$ and $G_{1}(\omega)$ satisfying $\forall \omega \in[0,+\infty)$

$$
\begin{aligned}
& N_{11}(\jmath \omega) D_{1}(\omega) N_{11}^{*}(\jmath \omega)+ \\
& \jmath\left(G_{1}(\omega) N_{11}^{*}(\jmath \omega)-N_{11}(\jmath \omega) G_{1}(\omega)\right) \leqslant D_{1}(\omega)
\end{aligned}
$$

\section{B. Validation procedure}

Then, the robust stability margin considering errors of estimation is dealt with. Let $\alpha_{m}$ be this margin defined by :

$$
\begin{aligned}
& \alpha_{m}=\max \left\{r \in \mathbb{R}^{+} \mid \forall \Delta \in B o, \forall \Delta_{\delta} \in r B_{\delta} o,\right. \\
& \text { the transfer matrix } p=\mathcal{F}_{u}\left(N(s), \operatorname{diag}\left(\tilde{\Delta}, \Delta_{\delta}\right)\right) d \\
& \text { is stable. }\}
\end{aligned}
$$

This margin allows to determine the maximal magnitude that the error of estimation can reach without causing the instability of the closed loop. However, the computation of $\alpha_{m}$ is difficult because the incertainties considered are both time invariant $(\tilde{\Delta})$ and time varying $\left(\Delta_{\delta}\right)$. To deal with them, the Robust Feedforward Design Toolbox (RFDT)[12] is used. Thanks to it, a lower bound of $\alpha_{m}$ is determined. Futhermore, for a margin of stability $\alpha=(1-\epsilon) \alpha_{m}(\epsilon>0$ given), one computes a guraranteed value of the worst case induced $L_{2}$ norm of the time varying uncertain transfer matrix, i.e a value $\gamma$ such that $\forall \tilde{\Delta} \in B o$, and $\forall \Delta_{\delta} \in \alpha B_{\delta} O$,

$$
\left\|\mathcal{F}_{u}\left(N(s), \operatorname{diag}\left(\tilde{\Delta}, \Delta_{\delta}\right)\right)\right\|_{i L_{2}} \leqslant \gamma
$$

The algorithms of this toolbox are based on the next proposition.

Proposition 4: Let $\Phi=\operatorname{diag}\left(\tilde{\Delta}, \Delta_{\delta}, \Delta_{c}\right)$, where $\Delta_{c} \in$ $\mathbb{C}^{m_{d} \times r_{p}}$ is an unstructured complex matrix. Let $D=D^{*}>0$ and $G=G^{*}$ be scaling matrices such that for all $\Phi$ having the indicated pattern they satisfy $D \Phi=\Phi D$ and $G \Phi=\Phi^{*} G$. The matrices $D$ and $G$ are divided as $D=$ $\operatorname{diag}\left(D_{1}, D_{2}, I\right)$ and $G=\operatorname{diag}\left(G_{1}, G_{2}, 0\right)$ in order to correspond to the decomposition of $\Phi$ in $\Delta, \Delta_{\delta}$ and $\Delta_{c}$. $D_{2}$ and $G_{2}$ are constant, while $D_{1}$ and $G_{1}$ vary with the frequency. Let $P=\Lambda(\alpha, \gamma) N$, with $\Lambda(\alpha, \gamma)=\operatorname{diag}\left(I_{r_{1}}, \frac{1}{\alpha} I_{r_{2}}, \frac{1}{\gamma} I_{r_{p}}\right)$, $r_{1}=q_{1}+\ldots+q_{n}$ and $r_{2}=k_{1}+\ldots+k_{n}$. If $D(\omega)$ and $G(\omega)$ exist such that $\forall \omega \in[0,+\infty)$

$$
\begin{aligned}
& P(\jmath \omega) D(\omega) P^{*}(\jmath \omega)+ \\
& \jmath\left(G(\omega) P^{*}(\jmath \omega)-P(\jmath \omega) G(\omega)\right) \leqslant D(\omega)
\end{aligned}
$$


then $\forall \Delta \in B o$, and $\forall \Delta_{\delta} \in \alpha B_{\delta} o$, the scheme of figure 4 is stable and (9) holds.

\section{THE ESTIMATOR}

Let $(A(\theta), B(\theta), I, 0)$ be a state space representation of $H(s, \theta)$ whose states are measured. The derivative of the state $x$ is rebuilt through a band-pass filter $\frac{s}{D(s)}$. Noting $\dot{x}_{f}=\left[\frac{s}{D(s)}\right] x$, it's supposed that $x_{f}=\left[\frac{1}{D(s)}\right] x$, and $u_{f}=\left[\frac{1}{D(s)}\right] u$. Then :

$$
\dot{x}_{f}=A x_{f}+B u_{f}
$$

So, assuming that matrices $A$ and $B$ are affine with respect to $\theta$, it's possible to rewrite (11) as

$$
\beta(t)=\theta^{T} \phi(t)
$$

where $\beta(t)$ and $\phi(t)$ are measured vector signals.

To obtain an estimation of $\theta$, a recursive least squares estimator is used. To be coherent with the implementation of this estimator, it is considered to be discrete. Thus, the time variable $t$ is considered to be in $\mathbb{N}$. The recursive least squares algorithm is given by :

$$
\begin{array}{r}
e(t)=\hat{\theta}^{T}(t) \phi(t)-\beta(t) \\
\hat{\theta}(t+1)=\hat{\theta}(t)-\frac{P(t) \phi(t) e^{T}(t)}{1+\phi^{T}(t) P(t) \phi(t)} \\
P(t+1)=P(t)-\frac{P(t) \phi(t) \phi(t)^{T} P(t)}{1+\phi^{T}(t) P(t) \phi(t)}
\end{array}
$$

with $P(0)>0$. If the plant input is exciting enough then $\lim _{t \rightarrow+\infty} \hat{\theta}(t) \rightarrow \theta$.

In futur works, slowly time varying parameters will be taken into account. In order to deal with these variations, the algorithm of recursive least squares can be modified to add a forgetting factor [17], [18], [14] or to initialize the matrix of covariance $P(t)$. But to ensure a good working order of the estimator on-line, algorithms without inversion or initialization of the covariance matrix will be favoured.

\section{ApPliCATION}

A linearised longitudinal model of a transport aircraft is considered. This model is extracted from the IMMUNE (Intelligent Monitoring and Managing of UNexpected Events) project in collaboration with Onera and DLR. The model considered is a transport aircraft of $160 \mathrm{~T}$. The linearization is realized during the cruise phase, at the height of $39000 \mathrm{ft}$ and with a number of Mach of 0.83 . Only the angle of attack $(\alpha)$ and the pitch rate $(q)$ are considered. The right and left elevators move together. The model is defined by the following equations

$$
\left\{\begin{array}{c}
\dot{\alpha}=Z_{\alpha} \alpha+Z_{q} q+Z_{u} u \\
\dot{q}=M_{\alpha} \alpha+M_{q} q+M_{u} u
\end{array}\right.
$$

The natural frequency of longitudinal mode is given by $\omega_{p}^{2}=Z_{\alpha} M_{q}-Z_{q} M_{\alpha}$. Considering nominal values of the model, it is noticed that $\left|\frac{Z_{\alpha} M_{q}}{Z_{q} M_{\alpha}}\right| \simeq 0.2$. Consequently, the term $Z_{q} M_{\alpha}$ is dominant, and as $Z_{q}$ is very close to 1 and doesn't vary much in practice, only variations of $M_{\alpha}$ will be considered. By the same way, $Z_{u}$ is close to 0 and doesn't vary much in practice, only variations of $M_{u}$ will be considered. Thus the vector of parameters $\theta$ to be estimated is $\theta=\left[M_{\alpha}, M_{u}\right]^{T}$. To move the elevator, a first order actuator is used $A c t(s)=\frac{1}{1+0.1 s}$, and the variables $\alpha, q$ and $\int \alpha$ are used by feedback. The scheme of figure 5 is obtained.

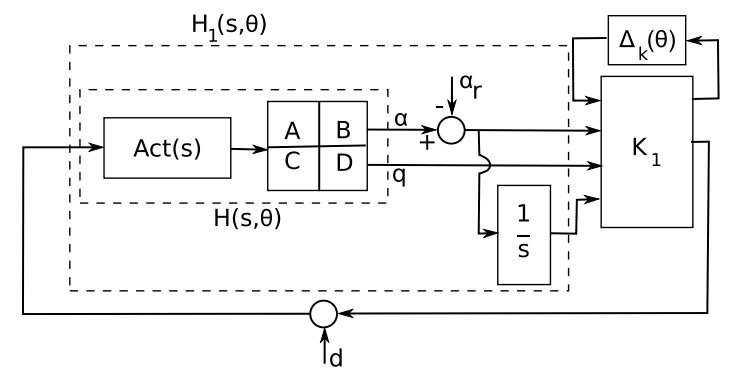

Fig. 5. Scheme used to design the LFT gain.

\section{A. Synthesis}

First of all, the synthesis is realized on the following worstcase model

$$
\left\{\begin{array}{l}
\dot{\alpha}=q \\
\dot{q}=M_{\alpha} \alpha+\eta
\end{array}\right.
$$

with the normalized input $\eta=M_{u} u$. In order to keep the same frequency for open and closed loops eigenvalues, an affine approximation of $M_{\alpha}$ with respect to $\omega_{p}$ is realized

$$
\omega_{p}=a \times M_{\alpha}+b
$$

with fixed $a$ and $b$. As there are three outputs, no more than three eigenvalues can be assigned.

$$
\begin{array}{r}
\lambda_{1}\left(M_{\alpha}\right)=\left(-\xi_{1}+\jmath \sqrt{1-\xi_{1}^{2}}\right) \omega_{p} \\
\lambda_{2}\left(M_{\alpha}\right)=-\xi_{1} \omega_{p}
\end{array}
$$

are chosen with the damping ratio $\xi_{1}=0.7 . \lambda_{2}\left(M_{\alpha}\right)$ is the closed loop value of the integrator pole. Then an LFT gain is designed with the function fb_sched of the Linear Fractional Representation Toolbox (LFRT) [19]. The LFT design model (17) depends only on the scheduling parameter $M_{\alpha}$. A controller $K_{1}\left(M_{\alpha}\right)$ is synthesized. The final controller between $(\alpha, q)$ and $(u)$ is obtained with the following formula

$$
\mathcal{F}_{u}\left(K_{\text {aug }}(s), \Delta_{k}(\theta)\right)=\frac{1}{M_{u}} \times K_{1}\left(M_{\alpha}\right) \times\left(\begin{array}{cc}
1 & 0 \\
0 & 1 \\
\frac{1}{s} & 0
\end{array}\right)
$$

The parameter $M_{\alpha}$ is repeated 8 times in the LFT controller, and the parameter $M_{u}$ appears only one time.

\section{B. Results of validation}

Following the procedure described in IV-A, the closed loop of figure 4 is obtained. A lower bound of its robust stability margin is computed as $\alpha_{m} \geqslant 16 \%$. In other words, if the relative estimation error doesn't exceed $16 \%$ of the parameter 
nominal value, the stability of the closed loop is ensured, even during the transient states. Futhermore, the guaranteed value $\gamma(9)$ of the induced $L_{2}$ norm of the transfer matrix between $d$ and $p=[\alpha, q]^{T}$ is visualized on figure 6 as a function of the size $\alpha$ of the estimation error.

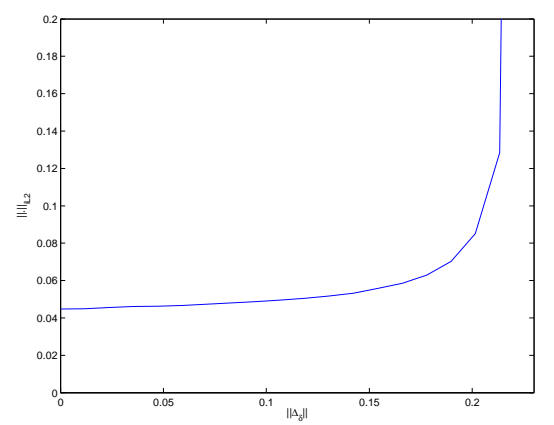

Fig. 6. Variation of the upper bound of the induced norm with the minimal relative estimation error $\Delta_{\delta}$ ensuring the stability.

\section{Time simulation}

As indicated in the paragraph $\mathrm{V}$, the estimation of parameters is obtained from measurements of the state $x=[\alpha, q]^{T}$ and the input $u$ of the system. These signals are filtered using the second order filter $\left[\frac{1}{D(s)}\right]$ with

$$
D(s)=\left(1+\tau_{1} s\right)\left(1+\tau_{2} s\right)
$$

and $\tau_{1}=5 \times 10^{-2}, \tau_{2}=\frac{\tau_{1}}{100}$.

The estimator is discrete, and its sampling interval is $0.01 \mathrm{~s}$. From equation (16), assuming $\phi(t)=\left[\alpha_{f}, u_{f}\right]^{T}$ and $\beta(t)=\dot{q}_{f}-M_{q} q_{f}$, one obtains $\beta(t)=\theta^{T} \phi(t)$. Then, using the recursive least squares algorithm described by equations $(13,14,15)$, an estimation $\hat{\theta}$ is obtained.

The time simulation scheme is described by figure 7 . Noting $\theta_{0}=\left[M_{\alpha 0}, M_{u 0}\right]^{T}$ the nominal values of the

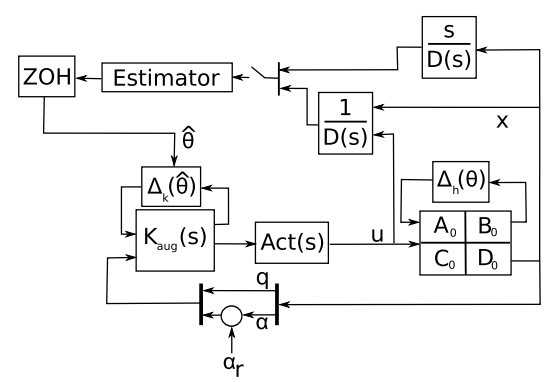

Fig. 7. Scheme of time simulation. ( $\mathrm{ZOH}=\mathrm{Zero}-$ Order Hold)

parameters, $\theta$ was chosen such that $M_{\alpha}=1.01 \times M_{\alpha 0}$, $M_{u}=1.7 \times M_{\alpha 0}$. The initial relative estimation error was set such that $\delta \theta=\left[0.06 \times M_{\alpha 0},-0.16 \times M_{u 0}\right]^{T}$.

The graph 8 shows the time responses to the input $\alpha_{r}$. The dashdot curves were obtained without the estimator, and the controller parameters are not adjusted while the solid ones correspond to the time responses with the estimator. The time responses when there is no estimation error are the dashed curves. But on this figure, the difference between the dashdot and dashed curves are not visible, because the estimator converges very quickly. The graph 9 shows the evolution of the estimation error.

It is noticed that the time response is improved when the estimator is added and is very close to the response without any estimation error. The pattern proposed guarentees good performances despite the initial unsuitable controller.
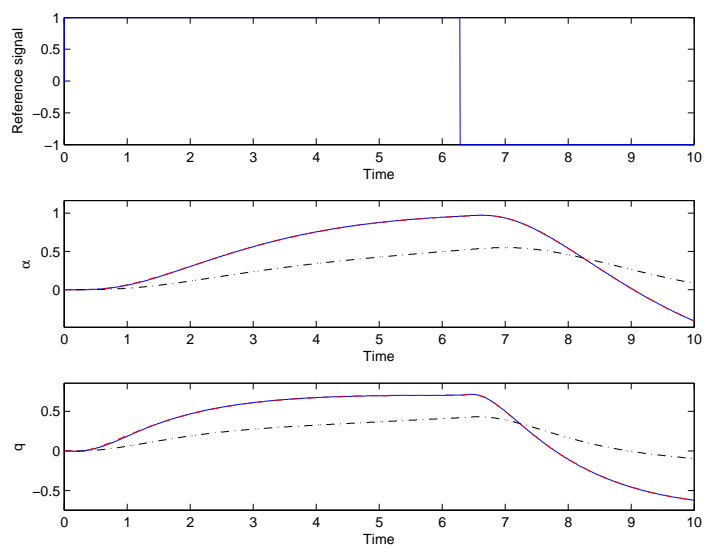

Fig. 8. Time responses of the simulation. The dashed curves are obtained when $\delta \theta=0$, the solid ones with an initial error estimation, and the dashdot ones were realized without estimator, so without any correction on the estimation error. The difference between the solid and the dashed curves is not visible.
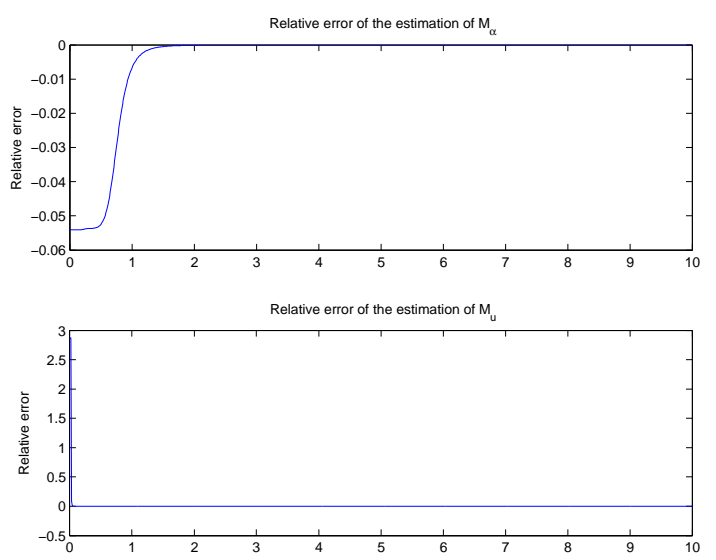

Fig. 9. Evolution of relative errors during the simulation with respect to the number of iterations.

\section{CONCLUSION}

In this article, robust and gain-scheduling analysis and design techniques were used to realize an adaptive controller. To analyse the stability and the performances of the closed loop, and to minimize the computational time of the controller adjustment, the plant and the controller are modeled in LFT form. This pattern allows the use of algorithms of modal design and validation through $\mu$-analysis. The approach is distinguished by the fact that the estimator doesn't appear explicitly in the analysis and synthesis schemes. Only bounds 
on the estimation errors are determined.

This technique is applied to control a transport aircraft. The model used is extracted from the IMMUNE project. A time domain simulation illustrates the nice transient properties of the adaptive scheme.

In the application, the scheme of the adaptive control gathers a continuous controller with a discrete estimator. An improvement of this scheme would be an adaptation of synthesis methods to a discrete plant in order to obtain a discrete controller, or an adaptation of analysis methods in order to take into account the hybrid nature of the scheme.

To apply this technique to the non-linear model of the transport aircraft of the IMMUNE project, another goal is to realize a "satisfactory" estimator of slowly time-varying parameters. Some tracks are given in the paragraph V.

\section{REFERENCES}

[1] C.J. Chien, K.C. Sun, A.C. Wu, et L.C. Fu. A robust MRAC using variable structure design for multivariable plants. Automatica, 32(6):833-848, 1996.

[2] T.C. Tsao et M.G. Safonov. Set theoretic adaptor control systems. Proceedings of the ACC, pages 3043-3047, 1993.

[3] T.C. Tsao et M.G. Safonov. Convex set theoretic adaptor control systems. Proceedings of the IEEE CDC, pages 582-584, 1993.

[4] T.C. Tsao et M.G. Safonov. Unfalsified direct adaptive control of a two-link robot arm. Proc. of the IEEE international Conference on Control Applications, pages 680-686, 1999.

[5] J.M. Krause, G. Stein, et P.P. Khargonekar. Sufficient conditions for robust performance of adaptive controllers with general uncertainty structure. Automatica, 28(2):277-288, 1992.

[6] P. Tomei. Robust adaptive control with arbitrary transient performance and disturbance attenuation. IEEE Transactions on Automatic Control, 44(3):654-658, 1999.

[7] Y. Zhang, C. Wen, et Y.C. Soh. Robust decentralized adaptive stabilisation of interconnected systems with guaranteed transient performance. Automatica, 36:907-915, 2000.

[8] S. Sastry. Nonlinear systems: analysis, stability and control. Springer Verlag, 1999.

[9] I. Barkana. Classical and simple adaptive control for nonminimum phase autopilot design. Journal of Guidance, Control and Dynamics, 28(4):631-638, 2005.

[10] G. Ferreres, V. Fromion, et M. M'Saad. Adaptive $H_{\infty}$ control using coprime factors and set-membership identification with application to a missile autopilot. Proceedings of the ACC, pages 698-702, 1995.

[11] J.H. Kelly et B.E. Ydstie. Adaptive $H_{\infty}$ control with application to systems with structural flexibility. IEEE Transactions on Automatic Control, 42(10):1358-1369, 1997.

[12] G. Ferreres et C. Roos. Robust feedforward design in the presence of LTI/LTV uncertainties. International Journal of Robust and Nonlinear Control, 17(14):1278-1293, september 2007.

[13] J.F. Magni. An LFT approach to robust gain scheduling. Proc. of the IEEE conference on Decision and Control, Seville, Spain, 2005.

[14] M E Salgado, G Goodwin, et R H Middleton. Modified least squares algorithm incorporating exponential forgetting and resetting. Int. J. Control, (47):477-491, 1988.

[15] J.F. Magni, A. Knauf, et C. Doll. Practical implementation of LFTscheduled feedback gains. Proc. of the Asian Control Conference, 2006.

[16] G. Ferreres. A practical approach to robustness analysis with aeronautical applications. Plenum Press / Kluwer Academic / Springer Verlag, 1999.

[17] G. Kreisselmeier. Stabilized least-squares type adaptive identifiers. Automatic Control, IEEE Transactions on, 35(3):306-310, Mar 1990

[18] M. Bodson. An adaptive algorithm with information-dependent data forgetting. American Control Conference, 1995. Proceedings of the, 5:3485-3489 vol.5, Jun 1995.

[19] J.F. Magni. Presentation of the Linear Fractional Representation Toolbox (LFRT). Proc. IEEE CACSD, Glasgow, UK, 2002. 